\title{
An analytic calculation method on air gap flux in permanent magnet brushless DC motor with ironless rotor
}

\author{
${ }^{1}$ Xinghua Wang, ${ }^{2}$ Yaolong Sheng and ${ }^{3}$ Shugang Zhao \\ 1,2,3 School of Electrical Engineering, Shangdong University, Jinan city, China \\ wangxinghua@sdu.edu.cn
}

\begin{abstract}
Keywords: ironless rotor, analytic calculation method, method of equivalent surfacecurrents, image method.

Abstract.An analytic calculation method about the air gap magnetic field of the high-speed permanent-magnet brushless DCmotor with ironless rotor is presented in this paper. Based on equivalent surfacecurrents method and image method, the mathematical model of the motor is presented. Then the analytical procession is deduced. And the calculated results of the method coincides with that of finite element method. It proves the analytic calculation method is exact and reliable.
\end{abstract}

\section{Introduction}

With the advantages of high power density, fast transient response and high operation efficiency, high-speed permanent magnet brushless DC motor has widely application prospects. In this paper, the high speed brushless DC motor applied in the small molecular pump is proposed as the research background. Due to its high speed,and the vacuumsurrounding environment, its capacity of heat dissipationis very poor. Therefore, the structure of ironless rotor is adopted for the less armature magnetic field andthe weak rotor eddy current loss. In order to get a reasonable and optimal ironless rotorstructure, the parameters of the motor must be optimized;Although the finite element method (FEM) can accurately calculate the performance of the motor, it is not conducive to the optimum design because of it'slow computational speed. However, the analytic calculation method is simple, parameterizable, fast and so on, so it is especially suitable for the optimization of the motor. The analytic calculation of the air gap flux has been deeply studied by scholars.

In confence [1], the analytic formulas of the unloaded air-gap flux in parallel-magnetized PM synchronous motor are derived from equivalent surfacecurrents. Considering the cogging of the motor, the air-gap relative ratio permeability function is constructed by Schwarz-Christoffel transformation. In confence[2], an exact analytic method is applied to compute air-gap field. The air gapfield is solved directly by the Laplasse equation or the Poisson equation of the three sub regions of the air gap, permanent magnet and slot, but the process is relatively complex. In confence[3],the superpositionprinciple is used to deduce the analytic expressions with the secondary air gap or no magnetic material inside the magnet. In this paper, equivalent mathematical model of ironless rotor structured high-speed permanent-magnet brushless DC motor is built based on equivalent surface current method and image method. An analytic expression for the air gap field of the motor is derived, and the analytic calculation results are compared with that of finite element method. Finally, it is proved that the analytic calculation method presented in this paper is correct and accurate. The method is suitable for optimization design of the motor with ironless rotor. 


\section{The principle of analytical calculation}

A. Equivalent surfacecurrents method of permanent magnet.In the analysis of electromagnetic field of permanent magnet motor, the following assumptions are generally adopted. 1)the core permeability of the stator $\mu_{\mathrm{Fe}}=\infty$;

2) the demagnetization curve of permanent magnetic material is linear;

3) the impact of the end of the motor is ignored;

4) the surface of the armature is smooth, and the equivalent air gap is considered.

The surface magnet pole is usually applied in the surface mounted permanent magnet motor. The 2 parallel magnetization magnetic pole of permanent magnet brushless DC motor is proposed as an example in this paper. The equivalent model of the equivalent surfacecurrents of the permanent magnetis shown in Fig. 1. The amplitude of surfacecurrents of the the permanent magnet face $\overline{A D}$ is the same as that of the face $\overline{B C}$, but they are in opposite direction. The current density can be expressed as $\mathrm{J}_{1}=\mathrm{H}_{\mathrm{c}} \sin \beta, \beta$ represents the current deviation from the center line of a point on the curve. Similarly, the current density along the $\overline{\mathrm{AB}}$ and $\overline{\mathrm{CD}}$ faces can be expressed as $J_{I}=H_{c} \cos \xi, \xi$ is $\pi$ in this paper.As the parallel magnetization is used, the magnetic flux density of $\overline{\mathrm{AB}}$ and $\overline{\mathrm{CD}}$ is 0 .
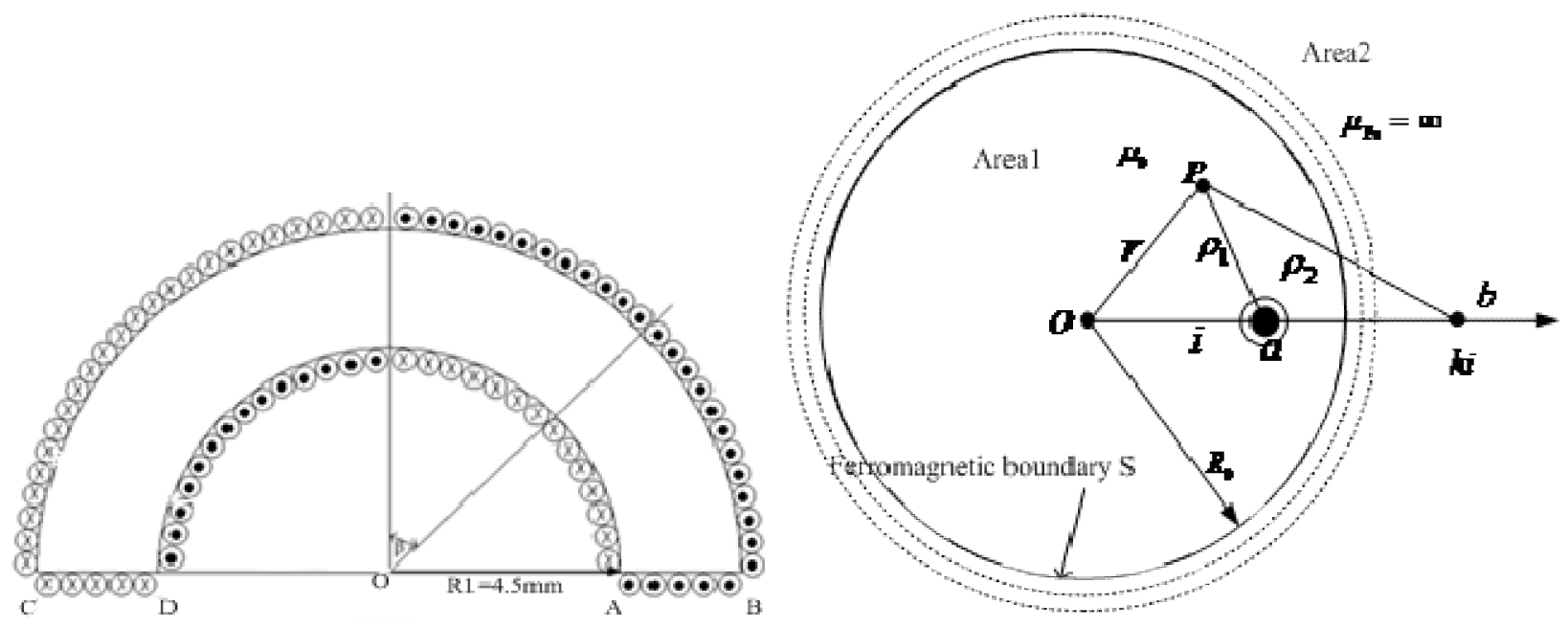

Fig. 1.Model of the equivalentFig. 2. The mirror current of the line surface currents of $\mathrm{N}$ pole current a in the ferromagnetic cylinder

B. Image method. As to the influence of the magnetic boundary, In this paper, the image method is used to analyze the equivalent surfacecurrents.As shown in Fig. 2, when the boundary interface is a long straight ferromagnetic cylinder and the line current is located in the ferromagnetic cylinder. The area within the radius of the cylinder is filled with the vacuum, $\mu_{1}=\mu_{0}$, while the magnetic permeability of ferromagnetic cylinders (area 2) $\mu_{2}=\mu_{\mathrm{Fe}}=\infty$; as well as $k=1, a b=R_{0}^{2}$. The line current $b$ is the mirror current of the line current $a$.

C. The model of permanent magnet motor with ironless rotor. Due to the rotor core and the 
shaft of the high speed motor are nonmagnetic material, they can be approximately equivalent to vacuum. According to the equivalent surface current method, the permanent magnet pole is equivalent to two annular surface currents, then the mirror current of them is deduced shown as Fig. 3. The mathematical model of the air gap field of the motor with ironless rotor is established. The mathematical model of the motor is divided into four solution areas, and the size of air gap flux is obtained by the superposition method.

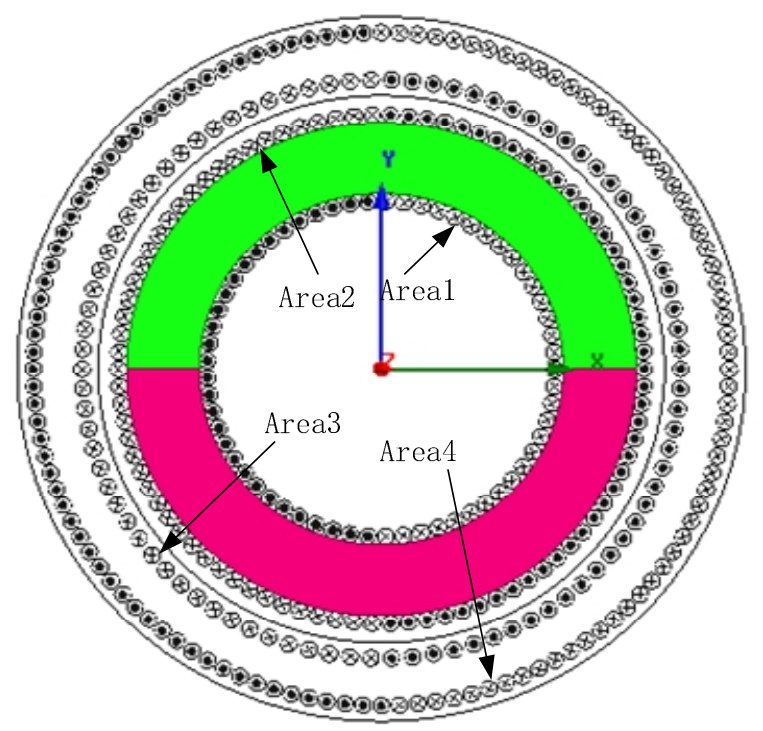

Fig. 3. Mathematical modelof the air flux

\section{The calculation of air flux}

Shown as Fig. 3, The radial air flux density is worked out by followingEq. 1 and Eq. 2.

$$
\begin{aligned}
& \mathrm{A}_{\mathrm{z}}=-\frac{\mu_{0} i}{4 \pi} \ln \rho^{2}+C \\
& \mathrm{~B}_{\mathrm{r}}=\frac{1}{r} \frac{\partial A_{z}}{\partial \theta}
\end{aligned}
$$

where $\rho$ represents the distance from any point on the surface current density ring to the point $\left(\mathrm{R}_{0} \cos \theta, R_{0} \sin \theta\right)$ on the air gap; $\theta$ represents the angle between the connecting line of the surface current ring and the point on the air gap circumference $\left(R_{0} \cos \theta, R_{0} \sin \theta\right)$ and the normal direction of the horizontal $\mathrm{X}$ axis; while $i=H_{c} \sin \beta R d \beta ; C$ is a constant.

Based on the above formula, the radial air flux density generated by 4 equivalent surfacecurrents rings at the same radius $R_{0}=7.5 \mathrm{~mm}$ can be calculated separately. The expression of the airradial flux density is obtained by processing the flux density expression: 


$$
\begin{aligned}
& B_{r 1}(\theta)=\frac{R_{1}}{r} \frac{\mu_{0} H_{c}}{2 \pi} \sin \theta\left(\frac{R_{0}^{2}+R_{1}^{2}}{R_{0} R_{1}} \beta-\frac{R_{0}^{2}-R_{1}^{2}}{R_{0} R_{1}} \arctan \left(\frac{\left(R_{0}^{2}+R_{1}^{2}\right) \tan \beta}{R_{0}^{2}-R_{1}^{2}}\right)\right)_{-\pi / 2+\theta}^{\pi / 2+\theta}(3) \\
& B_{r 2}(\theta)=-\frac{R_{2}}{r} \frac{\mu_{0} H_{c}}{2 \pi} \sin \theta\left(\frac{R_{0}^{2}+R_{2}^{2}}{R_{0} R_{1}} \beta-\frac{R_{0}^{2}-R_{2}^{2}}{R_{0} R_{1}} \arctan \left(\frac{\left(R_{0}^{2}+R_{2}^{2}\right) \tan \beta}{R_{0}^{2}-R_{2}^{2}}\right)\right)_{-\pi / 2+\theta}^{\pi / 2+\theta}(4) \\
& B_{r 3}(\theta)=-\frac{R_{2}}{r} \frac{\mu_{0} H_{c}}{2 \pi} \sin \theta\left(\frac{R_{0}^{2}+R_{3}^{2}}{R_{0} R_{3}} \beta-\frac{R_{0}^{2}-R_{3}^{2}}{R_{0} R_{3}} \arctan \left(\frac{\left(R_{0}^{2}+R_{3}^{2}\right) \tan \beta}{R_{0}^{2}-R_{3}^{2}}\right)\right)_{-\pi / 2+\theta}^{\pi / 2+\theta}(5) \\
& B_{r 4}(\theta)=\frac{R_{1}}{r} \frac{\mu_{0} H_{c}}{2 \pi} \sin \theta\left(\frac{R_{0}^{2}+R_{4}^{2}}{R_{0} R_{4}} \beta-\frac{R_{0}^{2}-R_{4}^{2}}{R_{0} R_{4}} \arctan \left(\frac{\left(R_{0}^{2}+R_{4}^{2}\right) \tan \beta}{R_{0}^{2}-R_{4}^{2}}\right)\right)_{-\pi / 2+\theta}^{\pi / 2+\theta} \\
& B_{r}(\theta)=B_{r 1}(\theta)+B_{r 2}(\theta)+B_{r 3}(\theta)+B_{r 4}(\theta)(7)
\end{aligned}
$$

As it shows, $R_{1}$ represents the outer radius of the rotor, $R_{2}$ represents the outer radius of the permanent magnet.

\section{The numerical result analysis}

In order to verify the accuracy of the analytic calculation method, A prototype motor showed as table 1 is presented. The air flux density is respectively worked out by above analytic calculation and FEM in this paper.

Table 1.Prototype parameters

\begin{tabular}{|l|l|l|l|}
\hline parameter & value & parameter & value \\
\hline Phase number & 3 & Inner radius of the stator [mm] & 16 \\
\hline Pole number & 2 & Outer radius of the rotor [mm] & 9 \\
\hline Slot number & 6 & Permanent magnet thickness [mm] & 2.3 \\
\hline Number of parallel branches & 1 & Permanent magnet material & $\begin{array}{l}\text { Bonded } \\
\text { NdFeB }\end{array}$ \\
\hline Outer radius of the stator [mm] & 40 & Magnetizing method & Parallel \\
\hline Length of iron core $[\mathrm{mm}]$ & 22 & Rated speed [rpm] & 72000 \\
\hline
\end{tabular}

Fig. 4 shows the comparison between analytical results and that of finite element method.As we can find out from the figure,the hollow part in the result of finite element analysis is caused by the stator slots. Therefore, neglecting the influence of stator slot, the two results are basically consistent with each other. It proves the analytic method presented in this paper is correct and exacet. 


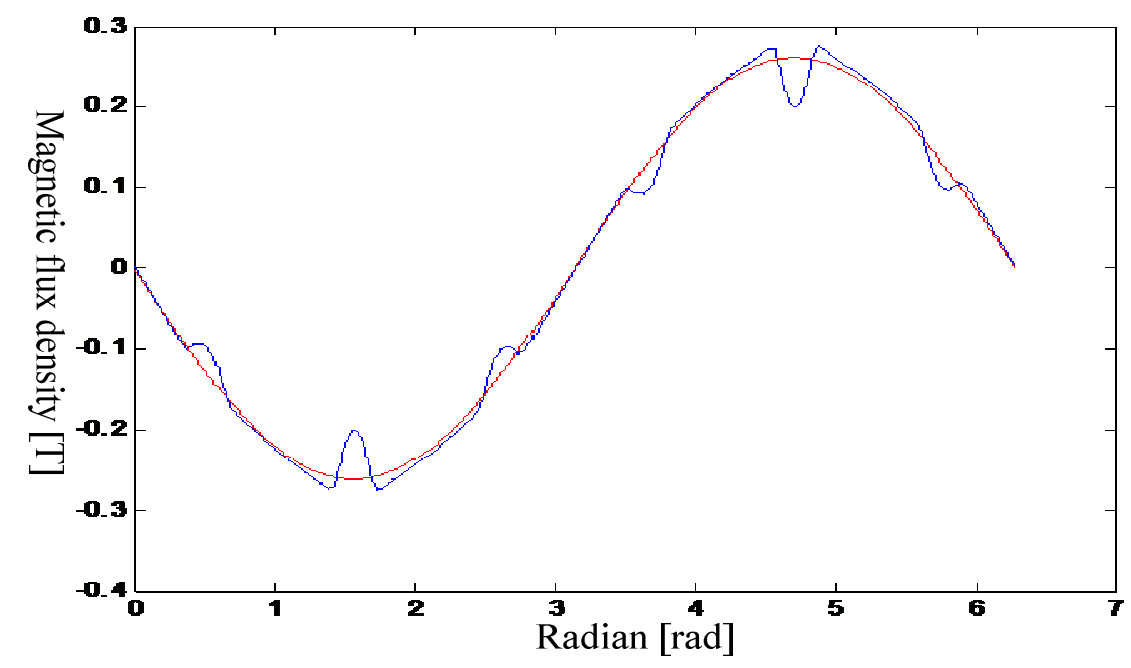

Fig. 4. Comparison between results of analytic method and FEM

\section{Conclusion}

The ironless rotorstructureis very effective for reducing rotoreddy current loss in high speed motors. The analytic calculation method of air flux is a powerful tool for the design and optimization of this kind of motor. The mathematical model of ironless rotor structure is derived based on equivalent surfacecurrents method and image method in this paper, and the air gap flux of the motor is calculated by it. The calculation results is fit with that of FEM, Itproves that the analytic calculation method of the air gap flux presented in this paper is correct and effective.

\section{Acknowledgements}

This work was financially supported byNational Key Scientific Apparatus Development of Special Item (2013YQ130429).

\section{References}

[1] Pan Yuanzhang, Rang Yuqi, and Huang Kefeng: Analytical Calculation of No-Looad Air-Gap Magnetic Field of Surface Permanent Magnet Synchronous Motor [J]. Explosion-proof Electric Machine, Vol. 47 (2012), No. 6, pp. 1-5

[2] Li Jiebao, Jing Libing, Zhou Xiaoyan, Zhang Yuejin: Exact Analytical Method for Surface-Mounted Permanent-Magnet Brushless Motors[J]. Transactions of China Electrotechnical Society, Vol. 27 (2012), No. 11, pp. 84-85

[3] Shen Jianxin, Chen Ligen: Analytical Calculation of Airgap Field in 2-Pole PermanentMagnet BrushlessMotor with Parallel-Magnetisation[J]. Electric Machines\&Control Application, Vol. 33(2006), No. 1, pp. 7-10 